\title{
Arzela-Ascoli Theorem for Demi-Linear Mappings
}

\author{
Qiang Lei ${ }^{1}$ and Aihong Chen ${ }^{2}$ \\ ${ }^{1}$ Department of Mathematics, Harbin Institute of Technology, Harbin 150001, China \\ ${ }^{2}$ Department of Applied Mathematics, Yanshan University, Yanshan 066004, China
}

Correspondence should be addressed to Qiang Lei; leiqiang@hit.edu.cn

Received 29 October 2013; Accepted 21 January 2014; Published 2 March 2014

Academic Editor: Donghai Ji

Copyright (C) 2014 Q. Lei and A. Chen. This is an open access article distributed under the Creative Commons Attribution License, which permits unrestricted use, distribution, and reproduction in any medium, provided the original work is properly cited.

The Arzela-Ascoli theorem for demi-linear mappings is established. Some conclusions about preserving totally bounded sets are also obtained.

\section{Introduction}

In 2009, Li et al. introduced the definition of demi-linear mappings which is a meaningful extension of linear operators [1]. They also established a new equicontinuity theorem and a uniform boundedness principle for demi-linear mappings. After that, many related theories (such as demi-linear duality, demi-distributions, and matrix transformations) are obtained for demi-linear mappings which can be found in [24].

Arzela-Ascoli theorem has a wide range of applications in many fields of mathematics. Many modern formulations of Arzela-Ascoli theorem have been obtained [5-10].

In this paper, we will give some results on Arzela-Ascoli theorem for the space of demi-linear mappings.

\section{Preliminary}

Let $X, Y$ be topological vector spaces over the scalar field $\mathbb{K}$ and $\mathscr{N}(X)$ the family of neighborhoods of $0 \in X$. Let

$$
=\left\{\gamma \in \mathbb{K}^{\mathbb{K}}: \lim _{t \rightarrow 0} \gamma(t)=\gamma(0)=0,|\gamma(t)| \geq|t| \text { if }|t| \leq 1\right\} .
$$

Definition 1 ([1, Definition 2.1]). A mapping $f: X \rightarrow Y$ is said to be demi-linear if $f(0)=0$ and there exist a $\gamma \in C(0)$ and a $U \in \mathcal{N}(X)$ such that $x \in X, u \in U$, and $t \in\{t \in \mathbb{K}$ : $|t| \leq 1\}$ yield $r, s \in \mathbb{K}$ for which $|r-1| \leq|\gamma(t)|,|s| \leq|\gamma(t)|$, and $f(x+t u)=r f(x)+s f(u)$.
We denote by $\mathscr{L}_{\gamma, U}(X, Y)$ the family of demi-linear mappings related to $\gamma \in C(0)$ and $U \in \mathcal{N}(X)$ and by $\mathscr{K}_{\gamma, U}(X, Y)$ the subfamily of $\mathscr{L}_{\gamma, U}(X, Y)$ satisfying the following property: if $x \in X, u \in U$, and $|t| \leq 1$, then $f(x+t u)=f(x)+s f(u)$ for some $s$ with $|s| \leq|\gamma(t)|$.

Note that, in general, $\mathscr{L}_{\gamma, U}(X, Y)$ is a large extension of $L(X, Y)$. For instance, if $\|\cdot\|: X \rightarrow[0,+\infty)$ is a norm, then $\|\cdot\| \in \mathscr{L}_{\gamma, X}(X, \mathbb{R})$ for every $\gamma \in C(0)$.

Definition 2. A family $\Gamma \subset Y^{X}$ is said to be equicontinuous at $x \in X$ if for every $W \in \mathcal{N}(Y)$, there is a $V \in \mathcal{N}(X)$ such that $f(x+V) \subset f(x)+W$ for all $f \in \Gamma$ and $\Gamma$ is equicontinuous on $X$ or, simply, equicontinuous if $\Gamma$ is equicontinuous at each $x \in X$.

By the proof of [1, Theorem 3.1], the following conclusion can be easily obtained.

Theorem 3. Let $\Gamma \subset \mathscr{L}_{\gamma, U}(X, Y)$. Then, $\Gamma$ is equicontinuous at 0 if and only If $\Gamma$ is equicontinuous on $X$.

By the proof of [1, Theorem 3.2] and Definition 2, we also have the following result.

Theorem 4. Let $\left\{f_{\alpha}\right\} \subset \mathscr{L}_{\gamma, U}(X, Y)$ and for every $x \in X$ we have $\lim f_{\alpha}(x)=f(x)$. Then, $f \in \mathscr{L}_{\gamma, U}(X, Y)$. If, in addition, $\left\{f_{\alpha}\right\}$ is equicontinuous, then $f$ is continuous.

Definition 5. A family $\Gamma \subset Y^{X}$ is said to be uniformly continuous if for any neighborhood $W$ of zero in $Y$, there exists a neighborhood $V$ of zero in $X$ such that $x_{1}-x_{2} \in V$ implies $f\left(x_{1}\right)-f\left(x_{2}\right) \in W$. 
Definition 6. Let $E$ be a nonempty set and $X$ a topological vector space, $F \subset X^{E}$. A topology $\tau$ on $E$ is said to be $(E, F)$ topology if there is an $\mathscr{F} \subset 2^{F}$ such that $\cup \mathscr{F}=F$ and $\tau$ is just the topology of uniform convergence on each $M \in \mathscr{F}$; that is, $u_{\alpha} \rightarrow u$ in $(E, \tau)$ if and only if for every $M \in \mathscr{F}$, $f\left(u_{\alpha}\right) \rightarrow f(u)$ uniformly with respect to $f \in M$.

Let $\sigma(E, F)$ be the weakest $(E, F)$-topology; that is, $u_{\alpha} \rightarrow$ $u$ in $(E, \sigma(E, F))$ if and only if $f\left(u_{\alpha}\right) \rightarrow f(u)$, for all $f \in F$.

Let $E, X$, and $F$ be as in Definition 6. For $x \in E$, define $x$ : $X^{E} \rightarrow X$ by $x(f)=f(x)$. Then, $E \subset X^{F}$ and by Definition 6 we have the $(F, E)$-topology on $F$. Also, we denote the weakest $(F, E)$-topology by $\sigma(F, E)$, namely, the topology of pointwise convergence. That is,

$$
f_{\alpha} \stackrel{\sigma(F, E)}{\longrightarrow} f \quad \text { in } F \text { iff } \forall x \in X, \quad \lim _{\alpha} f_{\alpha}(x)=f(x) .
$$

\section{Main Results}

Throughout this section, let $X$ and $Y$ be topological vector spaces on $\mathbb{C}$. Denote by $\mathscr{L}_{\gamma, U_{0}}(X, Y)$ the family of demi-linear mappings related to $\gamma \in C(0)$ and $U_{0} \in \mathcal{N}(X)$. For $U_{0} \in$ $\mathcal{N}(X)$, there is a balanced closed $U \in \mathcal{N}(X)$ such that $U \subset U_{0}$. Then, $\mathscr{L}_{\gamma, U_{0}}(X, Y) \subset \mathscr{L}_{\gamma, U}(X, Y)$. Without generality, we will assume that $U_{0}$ is balanced closed in $\mathscr{L}_{\gamma, U_{0}}(X, Y)$.

Recall that a set $S$ in a topological vector space is called totally bounded if for each neighborhood $U$ of zero there is a finite set $F$ with $S \subset F+U$.

Theorem 7. Let $f \in \mathscr{L}_{\gamma, U_{0}}(X, Y)$. If $f$ is continuous at zero, then $f$ preserves totally bounded sets.

Proof. Suppose that $B$ is a totally bounded subset of $X$. Since $Y$ is a topological vector space, for every balanced closed neighborhood $V \in \mathcal{N}(Y)$, there exists a balanced closed neighborhood $V_{1} \in \mathcal{N}(Y)$ such that $V_{1}+V_{1} \subset V$. For $V_{1}$, there is a $U \in \mathscr{N}(X)$ with $U \subset U_{0}$ such that

$$
f(U) \subset \frac{1}{1+|\gamma(1)|} V_{1}
$$

by the continuity of $f$ at zero. For $U$, there exists a finite subset $\left\{x_{1}, x_{2}, \ldots, x_{m}\right\}$ of $B$ such that $B \subset \bigcup_{i=1}^{m}\left(x_{i}+U\right)$; that is, for every $x \in B$ there exists an $i_{0}$ with $1 \leq i_{0} \leq m$ and $u \in U$ such that $x=x_{i_{0}}+u$. Then,

$$
f(x)=f\left(x_{i_{0}}+u\right)=r_{x} f\left(x_{i_{0}}\right)+s_{x} f(u),
$$

where $\left|r_{x}-1\right| \leq|\gamma(1)|$ and $\left|s_{x}\right| \leq|\gamma(1)|$. Let $A=\left\{f\left(x_{i}\right)\right.$ : $i=1, \ldots, m\}$ and $\widetilde{A}$ be the balanced hull of $A$. Obviously, the finite set $A$ is totally bounded and hence $\widetilde{A}$ is totally bounded. From

$$
\begin{aligned}
M & =\left\{r_{x} f\left(x_{i}\right): i=1, \ldots, m, x \in B\right\} \\
& =(1+|\gamma(1)|)\left\{\frac{r_{x}}{1+|\gamma(1)|} f\left(x_{i}\right): i=1, \ldots, m, x \in B\right\} \\
& \subset(1+|\gamma(1)|) \widetilde{A},
\end{aligned}
$$

it follows that $M$ is totally bounded and hence there exists a finite subset $\left\{y_{1}, y_{2}, \ldots, y_{n}\right\}$ of $Y$ such that

$$
M \subset\left\{y_{1}, y_{2}, \ldots, y_{n}\right\}+V_{1} .
$$

Then, from (3), (4), and (6), we have

$$
\begin{aligned}
f(x) & \in M+s_{x} f(U) \\
& \subset\left\{y_{1}, y_{2}, \ldots, y_{n}\right\}+V_{1}+\frac{s_{x}}{1+|\gamma(1)|} V_{1} \\
& \subset\left\{y_{1}, y_{2}, \ldots, y_{n}\right\}+V_{1}+V_{1} \\
& \subset\left\{y_{1}, y_{2}, \ldots, y_{n}\right\}+V
\end{aligned}
$$

for all $x \in B$. Thus, $f(B)$ is totally bounded.

Recall that a mapping with uniform continuity is also equicontinuous. The following theorem shows that the converse holds true for mappings in $\mathscr{K}_{\gamma, U_{0}}(X, Y)$.

Theorem 8. Let $\Gamma \subset \mathscr{L}_{\gamma, U_{0}}(X, Y)$. Then, $\Gamma$ is uniformly continuous if and only if $\Gamma$ is equicontinuous at zero.

Proof. We only need to prove the "if" part. For every $W \in$ $\mathcal{N}(Y)$, there exists a balanced closed $W_{1} \in \mathcal{N}(Y)$ with $W_{1} \subset$ $W$. For $(1 /|\gamma(1)|) W_{1} \in \mathscr{N}(Y)$, since $\Gamma$ is equicontinuous at zero, there is a $V \in \mathcal{N}(X)$ such that

$$
f(V) \subset\left(\frac{1}{|\gamma(1)|}\right) W_{1}
$$

for all $f \in \Gamma$. For arbitrary $x_{1}, x_{2} \in X$ with $x_{1}-x_{2} \in V$, we have

$$
f\left(x_{1}\right)=f\left(x_{2}+x_{1}-x_{2}\right)=f\left(x_{2}\right)+s_{f} f\left(x_{1}-x_{2}\right),
$$

where $\left|s_{f}\right| \leq|\gamma(1)|$ and $f \in \Gamma$. It follows that

$$
\begin{aligned}
f\left(x_{1}\right)-f\left(x_{2}\right) & =s_{f} f\left(x_{1}-x_{2}\right) \\
& \in s_{f} f(V) \\
& \subset s_{f}\left(\frac{1}{|\gamma(1)|}\right) W_{1} \\
& \subset|\gamma(1)|\left(\frac{1}{|\gamma(1)|}\right) W_{1} \\
& =W_{1} \subset W
\end{aligned}
$$

for all $f \in \Gamma$, which shows that $\Gamma$ is uniformly continuous.

Theorem 9 (Ascoli Theorem). Let $X, Y$ be topological vector spaces, $Y$ be Hausdorff, and $\mathscr{E}$ be the family of all compact subsets of $X$. Let $\tau_{\mathscr{E}}$ be the topology on $\mathscr{L}_{\gamma, U_{0}}(X, Y)$ of uniform convergence on each $K \in \mathscr{E}$. If $\Gamma \subset \mathscr{\mathscr { L }}_{\gamma, U_{0}}(X, Y)$ is equicontinuous and $\bar{\Gamma}^{\sigma\left(\mathscr{L}_{\gamma, U_{0}}(X, Y), X\right)}$ is compact, then $\bar{\Gamma}^{\tau_{\mathscr{g}}}$ is equicontinuous and compact.

Proof. Since $\Gamma$ is equicontinuous, for every balanced closed $V \in \mathcal{N}(Y)$, there exists a balanced closed $U \in \mathcal{N}(X)$ such 
that $f(U) \subset V$ for all $f \in \Gamma$. For every $f \in \bar{\Gamma}^{\tau_{\mathscr{g}}}$, there is a net $\left\{f_{\alpha}\right\} \subset \Gamma$ such that $f_{\alpha} \stackrel{\tau_{\mathscr{g}}}{\longrightarrow} f$. Since the singleton is compact, that is, $\{x\} \in \mathscr{E}$ for all $x \in X$, we have $\lim _{\alpha} f_{\alpha}(x)=f(x)$ for all $x \in X$. Then, by the balanced closed property of $V$, we have

$$
f(x)=\lim _{\alpha} f_{\alpha}(x) \in V, \quad \forall x \in U ;
$$

that is, $f(U) \subset V$. As $f$ runs over the set $\bar{\Gamma}^{\tau_{\mathscr{g}}}$, we have that $\bar{\Gamma}^{\tau_{\mathscr{\delta}}}$ is equicontinuous at zero and hence $\bar{\Gamma}^{\tau_{\mathscr{C}}}$ is equicontinuous on $X$ by Theorem 3 .

Let $\left\{f_{\alpha}: D\right\}$ be a net in $\bar{\Gamma}^{\tau_{\mathscr{g}}}$. Since the topology $\sigma\left(\mathscr{L}_{\gamma, U_{0}}(X, Y), X\right)$ is weaker than $\tau_{\mathscr{E}}$, we have $\bar{\Gamma}^{\tau_{\mathscr{E}}} \mathrm{C}$ $\bar{\Gamma}^{\sigma\left(\mathscr{L}_{\gamma, U_{0}}(X, Y), X\right)}$ and hence $\left\{f_{\alpha}: D\right\}$ is also a net in $\bar{\Gamma}^{\sigma\left(\mathscr{L}_{\gamma, U_{0}}(X, Y), X\right)}$. Since $\bar{\Gamma}^{\sigma\left(\mathscr{L}_{\gamma, U_{0}}(X, Y), X\right)}$ is compact, the net $\left\{f_{\alpha}\right.$ : $D$ \} has a convergent subnet of $\left\{f_{\alpha}: D\right\}$, which we also denote by $\left\{f_{\alpha}: D\right\}$. Let $f_{0}$ be the limit of $\left\{f_{\alpha}: D\right\}$ in $\bar{\Gamma}^{\sigma\left(\mathscr{L}_{\gamma, U_{0}}(X, Y), X\right)}$; that is,

$$
\lim _{\alpha} f_{\alpha}(x)=f_{0}(x), \quad \forall x \in X .
$$

Then, we have $f_{0} \in \mathscr{L}_{\gamma, U_{0}}(X, Y)$ and $f_{0}$ is continuous by Theorem 4

To prove $\bar{\Gamma}^{\tau_{\mathscr{g}}}$ is compact, we only need to prove $f_{\alpha} \stackrel{\tau_{\mathscr{g}}}{\longrightarrow} f_{0}$. Assume this is not true; then, there exists a $K \in \mathscr{E}$ such that $f_{\alpha}(x) \rightarrow f_{0}(x)$ is not uniform for $x \in K$. Then, there are $V \in \mathcal{N}(Y)$, increasing sequence $\left\{\alpha_{k}\right\} \subset D$, and $\left\{x_{k}\right\} \subset K$ such that

$$
f_{\alpha_{k}}\left(x_{k}\right)-f_{0}\left(x_{k}\right) \notin V .
$$

For $V$, there exists a balanced $V_{1} \in \mathcal{N}(Y)$ such that $V_{1}+$ $V_{1}+V_{1} \subset V$. Since $K$ is compact, the sequence $\left\{x_{n}\right\}$ has a convergent subnet $\left\{x_{n_{\delta}}\right\}$ with $x_{n_{\delta}} \rightarrow x_{0} \in K$. For $V_{1}$, there exists a $U \in \mathcal{N}(X)$ such that

$$
f\left(x_{0}+U\right) \subset f\left(x_{0}\right)+V_{1}, \quad \forall f \in \bar{\Gamma}^{\tau_{\xi}}
$$

by the equicontinuity of $\bar{\Gamma}^{\tau_{\mathscr{\tau}}}$. Then,

$$
f_{\alpha}\left(x_{0}+U\right) \subset f_{\alpha}\left(x_{0}\right)+V_{1}, \quad \forall \alpha \in D .
$$

For $U$, there exists a $\delta_{0}$ such that $x_{n_{\delta}} \in x_{0}+U$, for all $\delta \geq \delta_{0}$. Then,

$$
f_{\alpha}\left(x_{n_{\delta}}\right) \in f_{\alpha}\left(x_{0}\right)+V_{1}, \quad \forall \alpha \in D, \forall \delta \geq \delta_{0} .
$$

Since $f_{0}$ is continuous, there is a $\delta_{1} \geq \delta_{0}$ such that

$$
f_{0}\left(x_{n_{\delta}}\right) \in f_{0}\left(x_{0}\right)+V_{1}, \quad \forall \delta \geq \delta_{0} .
$$

Since $f_{\alpha}\left(x_{0}\right) \rightarrow f_{0}\left(x_{0}\right)$, there exists an $\alpha_{0}$ such that

$$
f_{\alpha}\left(x_{0}\right) \in f_{0}\left(x_{0}\right)+V_{1}, \quad \forall \alpha \geq \alpha_{0} .
$$

Then, (16) and (18) imply that

$$
\begin{array}{r}
f_{\alpha}\left(x_{n_{\delta}}\right) \in f_{\alpha}\left(x_{0}\right)+V_{1} \subset f_{\alpha}\left(x_{0}\right)+V_{1}+V_{1}, \\
\forall \delta \geq \delta_{0}, \quad \forall \alpha \geq \alpha_{0} .
\end{array}
$$

It follows from (17) that

$$
\begin{aligned}
& f_{\alpha}\left(x_{n_{\delta}}\right)-f_{0}\left(x_{n_{\delta}}\right) \in f_{0}\left(x_{0}\right)+V_{1}+V_{1}-f_{0}\left(x_{0}\right)-V_{1} \\
& \quad=V_{1}+V_{1}-V_{1} \subset V, \quad \forall \delta \geq \delta_{1}, \forall \alpha \geq \alpha_{0} .
\end{aligned}
$$

This contracts (13) and hence $f_{\alpha} \stackrel{\tau_{\mathscr{g}}}{\longrightarrow} f_{0}$, which yields the desired result.

In fact, the converse of Theorem 9 is also true. The following theorem gives the Arzela Theorem for demi-linear mappings.

Theorem 10 (Arzela Theorem). Let $X, Y$ be topological vector spaces, $Y$ be Hausdorff, and $\mathscr{E}$ be the family of all compact subsets of $X$. Let $\tau_{\mathscr{G}}$ be the topology on $\mathscr{L}_{\gamma, U_{0}}(X, Y)$ of uniform convergence on each $K \in \mathscr{E}$. If $\Gamma$ is a family of continuous mappings in $\mathscr{L}_{\gamma, U_{0}}(X, Y)$ and $\bar{\Gamma}^{\tau_{\mathscr{g}}}$ is compact, then

(I) $\Gamma$ is equicontinuous;

(II) $\bar{\Gamma}^{\sigma\left(\mathscr{L}_{\gamma, U_{0}}(X, Y), X\right)}$ is compact.

Proof. For conclusion (I), we just need to prove that $\Gamma$ is equicontinuous at zero. Suppose that $\Gamma$ is not equicontinuous at zero; that is, there exists a $V \in \mathcal{N}(Y)$ such that for every $U \in \mathcal{N}(X)$ there are $x_{U} \in U$ and $f_{U} \in \Gamma$ such that

$$
f_{U}\left(x_{U}\right) \notin V .
$$

Since $\mathcal{N}(X)$ is directed by the relation " $\subset$," we have that $\left\{x_{U}\right.$ : $\mathcal{N}(X)\}$ and $\left\{f_{U}: \mathcal{N}(X)\right\}$ are nets, respectively, in $X$ and $\mathscr{L}_{\gamma, U_{0}}(X, Y)$. Since $\left\{f_{U}: U \in \mathcal{N}(X)\right\} \subset \Gamma$ and $\bar{\Gamma}^{\tau_{\mathscr{F}}}$ is compact, the net $\left\{f_{U}: \mathcal{N}(X)\right\}$ has a convergent subnet, which we also denote by $\left\{f_{U}: \mathcal{N}(X)\right\}$. Let $f_{0}$ be the limit of $\left\{f_{U}: \mathscr{N}(X)\right\}$ in $\bar{\Gamma}^{\tau_{\mathscr{F}}}$; that is, for each $K \in \mathscr{E}$ we have $f_{U}(x) \rightarrow f_{0}(x)$ uniformly for $x \in K$. Obviously,

$$
\lim _{U} x_{U}=0
$$

and hence $\left\{x_{U}: U \in \mathscr{N}(X)\right\}$ is compact; that is, $\left\{x_{U}: U \in\right.$ $\mathcal{N}(X)\} \in \mathscr{E}$. Therefore, we have $f_{U}(x) \rightarrow f_{0}(x)$ uniformly for $\left\{x_{U}: U \in \mathcal{N}(X)\right\}$. For $V$, there exists a $V_{1} \in \mathcal{N}(Y)$ such that

$$
V_{1}+V_{1} \subset V .
$$

Then for $V_{1}$, there exists a $U_{1} \in \mathcal{N}(X)$ such that

$$
f_{U}(x)-f_{0}(x) \in V_{1}, \quad \forall U \subset U_{1}, \quad \forall x \in\left\{x_{V}: V \in \mathcal{N}(X)\right\} .
$$

It follows that

$$
f_{U}\left(x_{U}\right)-f_{0}\left(x_{U}\right) \in V_{1}, \quad \forall U \subset U_{1} .
$$

By (22) and the continuity of $f_{0}$, for $V_{1}$, there is a $U_{2} \in \mathcal{N}(X)$ such that

$$
f_{0}\left(x_{U}\right) \in V_{1}, \quad \forall U \subset U_{2} .
$$


From (23), (25), and (26), we have

$$
f_{U}\left(x_{U}\right)=\left(f_{U}\left(x_{U}\right)-f_{0}\left(x_{U}\right)\right)+f_{0}\left(x_{U}\right) \in V_{1}+V_{1} \subset V
$$

which is in contradiction with (21) and hence the desired result holds.

For conclusion (II), we will prove that $\bar{\Gamma}^{\sigma\left(\mathscr{L}_{\gamma, U_{0}}(X, Y), X\right)}=$ $\bar{\Gamma}^{\tau_{\mathscr{g}}}$. And we just need to show that $\bar{\Gamma}^{\sigma\left(\mathscr{L}_{\gamma, U_{0}}(X, Y), X\right)} \subset \bar{\Gamma}^{\tau_{\mathscr{g}}}$. For each $f \in \bar{\Gamma}^{\sigma\left(\mathscr{L}_{\gamma, U_{0}}(X, Y), X\right)}$, there exists a net $\left\{f_{\alpha}\right\}$ in $\Gamma$ such that $\lim _{\alpha} f_{\alpha}(x)=f(x)$, for all $x \in X$. We only need to prove that $f_{\alpha} \stackrel{\tau_{\mathscr{E}}}{\longrightarrow} f$. If this is not true, then there is a $K \in \mathscr{E}$ such that $f_{\alpha}(x) \rightarrow f(x)$ is not uniform for $x \in K$. Then, there are $V \in \mathcal{N}(Y)$, increasing sequence $\left\{\alpha_{k}\right\}$, and $\left\{x_{k}\right\} \subset K$ such that

$$
f_{\alpha_{k}}\left(x_{k}\right)-f\left(x_{k}\right) \notin V .
$$

Since $K$ is compact, there exist an $x_{0} \in K$ and a convergent subnet $\left\{x_{k_{\delta}}\right\}$ of $\left\{x_{k}\right\}$ such that $\lim _{\delta} x_{k_{\delta}}=x_{0}$. By the equicontinuity of $\Gamma$, the continuity of $f$, and (28), we have

$$
f_{k_{\delta}}\left(x_{0}\right)-f\left(x_{0}\right) \notin V \text {. }
$$

However,

$$
\lim _{\delta} f_{k_{\delta}}\left(x_{0}\right)=\lim _{\alpha} f_{\alpha}\left(x_{0}\right)=f\left(x_{0}\right)
$$

which leads a contradiction to (29) and hence the desired result holds.

Theorem 11. Let $X, Y$ be topological vector spaces and $X$ be of second category. If $\Gamma$ is a family of continuous mappings in $\mathscr{L}_{\gamma, U_{0}}(X, Y)$ and for every $x \in X$, the set $\{f(x): f \in \Gamma\}$ is totally bounded, then for every totally bounded subset $B$ of $X$, we have that $\{f(x): x \in B, f \in \Gamma\}$ is totally bounded.

Proof. Since totally bounded set is bounded, it follows that $\Gamma$ is equicontinuous on $X$ by the equicontinuity theorem [1, Theorem 3.1]. For every balanced closed $V \in \mathcal{N}(Y)$, there exists a balanced closed $V_{1} \in \mathcal{N}(Y)$ such that $V_{1}+V_{1} \subset V$. For $V_{1}$, there is a $U \in \mathcal{N}(X)$ with $U \subset U_{0}$ such that

$$
f(U) \subset \frac{1}{1+\gamma(1)} V_{1}, \quad \forall f \in \Gamma .
$$

For $U$, there exist $x_{1}, x_{2}, \ldots, x_{n} \in B$ such that $B \subset \bigcup_{i=1}^{n}\left(x_{i}+\right.$ $U)$; that is, for every $x \in B$, there exists an $i_{0}$ with $1 \leq i_{0} \leq n$ and $u \in U$ such that $x=x_{i_{0}}+u$. Then,

$$
f(x)=f\left(x_{i_{0}}+u\right)=r_{f} f\left(x_{i_{0}}\right)+s_{f} f(u),
$$

where $\left|r_{f}-1\right| \leq|\gamma(1)|,\left|s_{f}\right| \leq|\gamma(1)|$, and $f \in \Gamma$. Let $A=\left\{f\left(x_{i}\right): f \in \Gamma, i=1,2, \ldots, n\right\}$. Since for every $x \in X$, the set $\{f(x): f \in \Gamma\}$ is totally bounded, we see that $A$ is totally bounded. Let $\widetilde{A}$ be the balanced hull of $A$; that is, $\widetilde{A}=\bigcup_{|t| \leq 1} t A$. Then, $\widetilde{A}$ is totally bounded. It follows from

$$
\begin{aligned}
\left\{r_{f} A: f \in \Gamma\right\} & =(1+|\gamma(1)|)\left\{\frac{r_{f}}{1+|\gamma(1)|} A: f \in \Gamma\right\} \\
& \subset(1+|\gamma(1)|) \widetilde{A}
\end{aligned}
$$

that $\left\{r_{f} A: f \in \Gamma\right\}$ is totally bounded. Hence, there exist $y_{1}, y_{2}, \ldots, y_{m} \in Y$ such that

$$
\left\{r_{f} A: f \in \Gamma\right\} \subset\left\{y_{1}, y_{2}, \ldots, y_{m}\right\}+V_{1}, \quad \forall f \in \Gamma .
$$

Then, (31), (32), and (34) imply that

$$
\begin{aligned}
f(x) & =r_{f} f\left(x_{i_{0}}\right)+s_{f} f(u) \\
& \in r_{f} A+\frac{s_{f}}{1+|\gamma(1)|} V_{1} \\
& \subset\left\{y_{1}, y_{2}, \ldots, y_{m}\right\}+V_{1}+V_{1} \\
& \subset\left\{y_{1}, y_{2}, \ldots, y_{m}\right\}+V
\end{aligned}
$$

for all $x \in B$ and $f \in \Gamma$. Thus, $\{f(x): x \in B, f \in \Gamma\}$ is totally bounded.

\section{Conflict of Interests}

The authors declare that there is no conflict of interests regarding the publication of this paper.

\section{Acknowledgments}

This work is supported by National Natural Science Foundation of China (Project no. 11101108), China Postdoctoral Science Foundation (Project no. 2011M500646), and Natural Scientific Research Innovation Foundation in Harbin Institute of Technology (Project no. 2011008).

\section{References}

[1] R. Li, S. Zhong, and L. Li, "Demi-linear analysis. I. Basic principles," Journal of the Korean Mathematical Society, vol. 46, no. 3, pp. 643-656, 2009.

[2] R. Li, A. Chen, and S. Zhong, "Demi-linear duality," Journal of Inequalities and Applications, vol. 2011, article 128, 2011.

[3] R. Li and S. Zhong, " $l^{\infty}(x)-l^{p}(y)$ summability of mapping matrices," Taiwanese Journal of Mathematics, vol. 14, no. 6, pp. 2291-2305, 2010

[4] S. Zhong and R. Li, "Uniform boundedness principles for ordered topological vector spaces," Annals of Functional Analysis, vol. 2, no. 1, pp. 13-18, 2011.

[5] J. Collins and J. Zimmer, "An asymmetric Arzelà-Ascoli theorem," Topology and Its Applications, vol. 154, no. 11, pp. 23122322, 2007.

[6] H. Hanche-Olsen and H. Holden, "The Kolmogorov-Riesz compactness theorem," Expositiones Mathematicae, vol. 28, no. 4, pp. 385-394, 2010.

[7] R. Li, S. Zhong, and C. Swartz, "An improvement of the ArzelaAscoli theorem," Topology and Its Applications, vol. 159, no. 8, pp. 2058-2061, 2012.

[8] J. L. Kelley, General Topology, Springer, New York, NY, USA, 1991.

[9] R. Engelking, General Topology, Polish Scientific Publishers, Warsaw, Poland, 1977.

[10] J. R. Munkres, Topology: A First Course, Prentice-Hall, Englewood Cliffs, NJ, USA, 1975. 


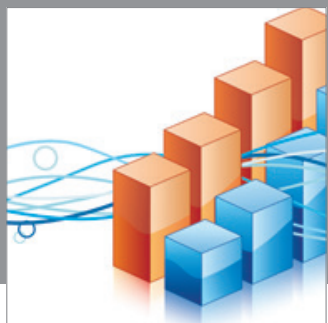

Advances in

Operations Research

mansans

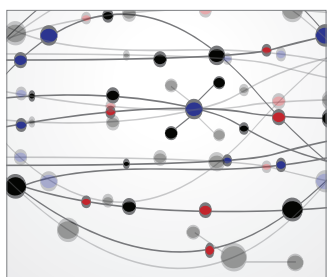

The Scientific World Journal
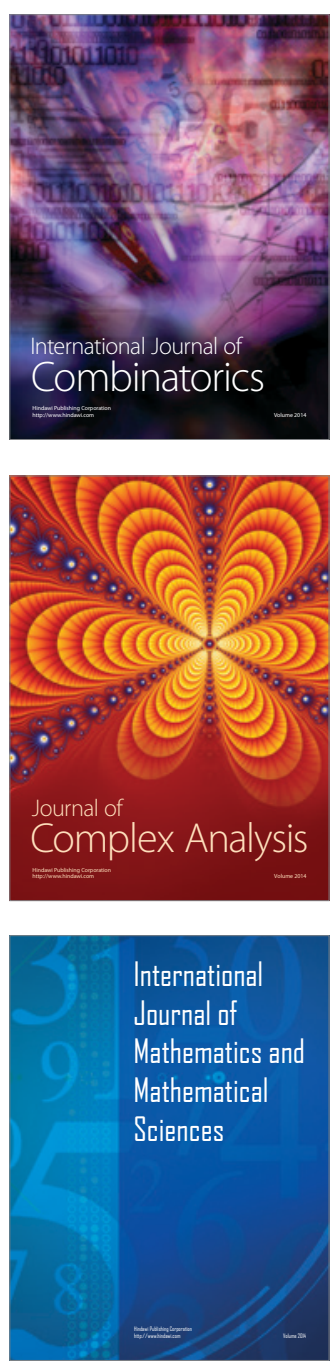
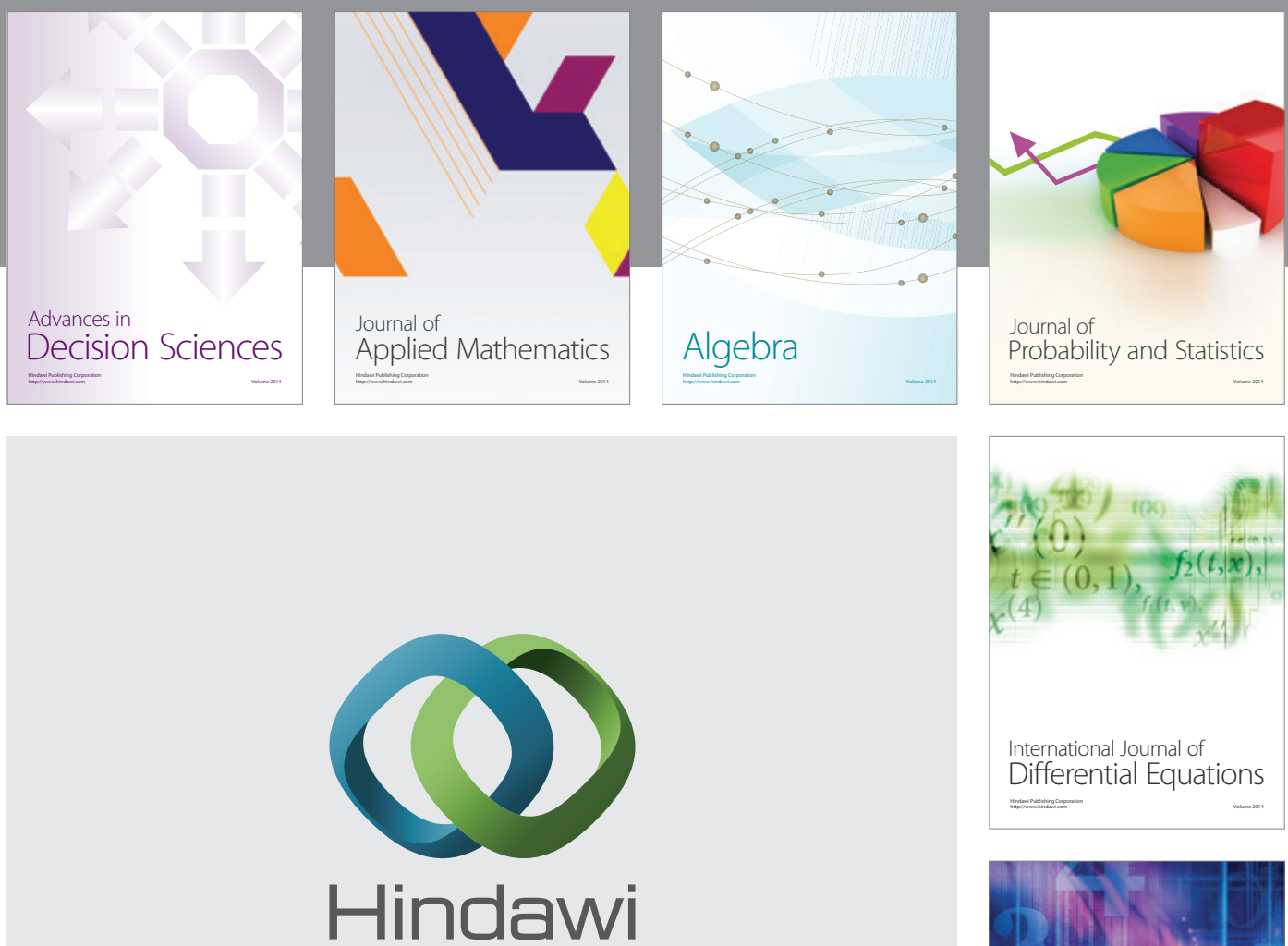

Submit your manuscripts at http://www.hindawi.com
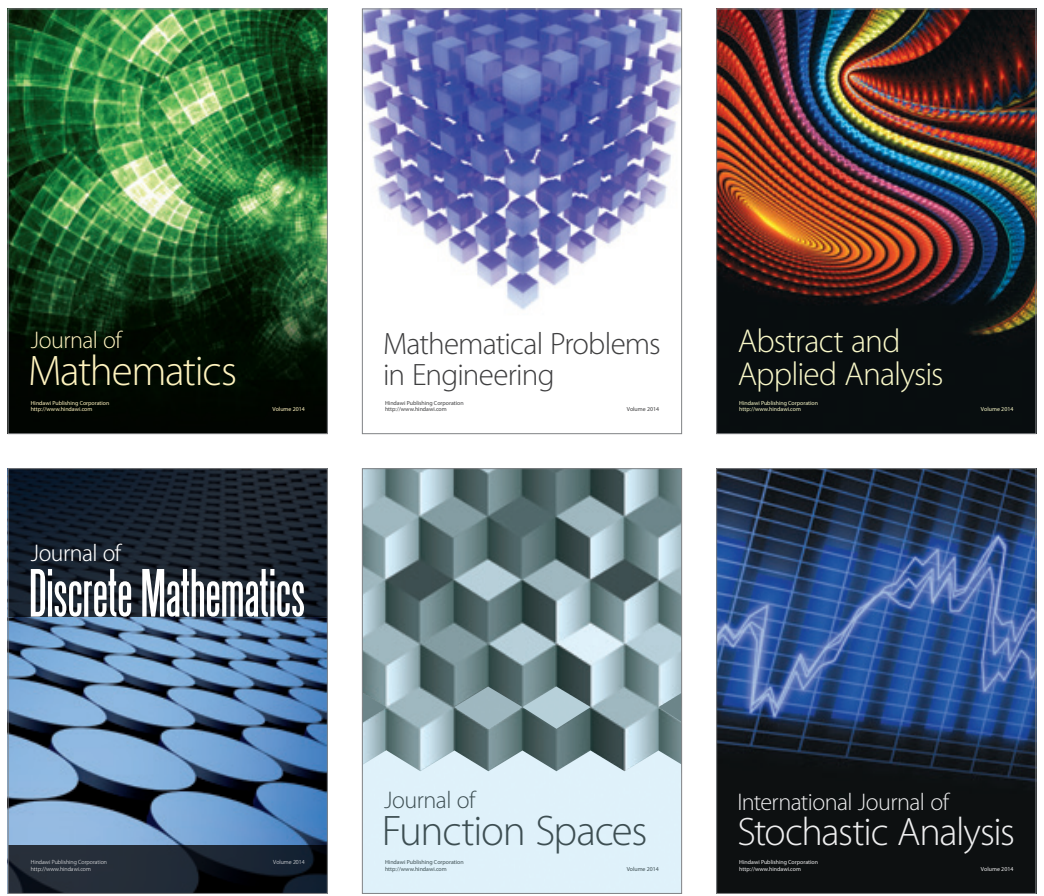

Journal of

Function Spaces

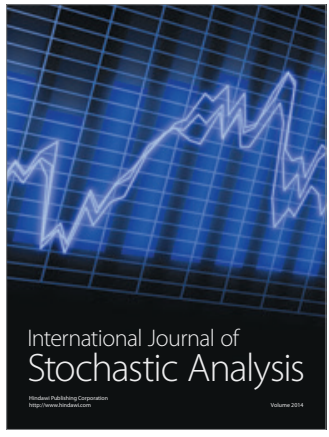

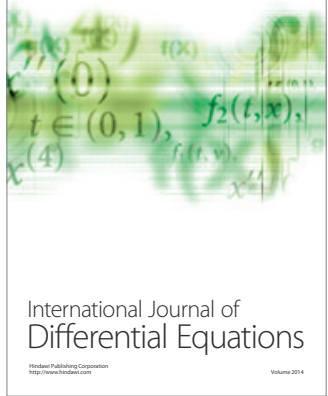
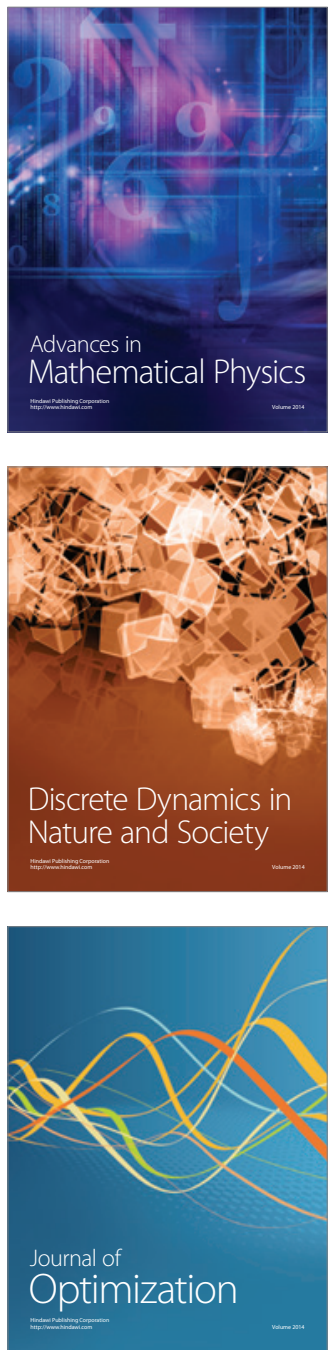